\title{
Comparison of Multisectoral approaches withstand alone Nutrition Specific Interventions to Address Malnutrition in Sanghar, Sindh
}

\section{Khan $\mathrm{MI}^{*}$ and Khalid R}

Save the children country office, Sindh, Pakistan

${ }^{*}$ Corresponding author: Khan MI, Save the children country office, Sindh, Pakistan, Tel: 00923339311750, E-mail: isakkhan9@gmail.com

Citation: Khan MI, Khalid R (2015) Comparison of Multisectoral approaches withstand alone Nutrition Specific Interventions to Address Malnutrition in Sanghar, Sindh. J Nutr Health Sci 2(4): 402. doi:

$10.15744 / 2393-9060.2 .402$

Received Date: August 26, 2015 Accepted Date: November 24, 2015 Published Date: November 25, 2015

\begin{abstract}
Background: Improved nutrition of an individual or community can contribute to increased productivity, improvement of the economic status of household or the community due to improved physical capacities, increased immunity against diseases and increased life expectancy. Cognitive development followed by improved school performance can also be achieved through quality and balanced nutrition. Poor nutrition status is leading to affect the physical capabilities, increased susceptibility to diseases, and economic losses due to poor physical and cognitive capabilities and loss of assets or wealth on the management of disease - contributing to cycle of poverty, malnutrition, and disease and vice versa. Nutrition sensitive and specific public policies are the only options to reduce poverty, improved health status of a society, and productivity of a nation as is suggested by various studies and movements like the Lancet Analysis of Maternal and Child Nutrition Series 2008 and the Scaling Up Nutrition (SUN) movement.

Save the Children in close collaboration and coordination with provincial and district department of health to implement emergency project "Pakistan Emergency Food Security Alliance" (PEFSA V) through supporting nutrition specific and nutrition sensitive interventions in the flood and drought affected district Sanghar, Sindh.

Methodology: The study is based on the assessment of treatment outcome of the children among nutrition specific interventions (Non - FSL Support Group) and the same among the group of the children whose families also received additional support to improve food security of the household (FSL Support Group). The data has been obtained from the programme data base and the secondary data was analyzed with SPSS 20.0 .

Results: The results of the comparative cross sectional observations shows that there is a slight faster weight gain among the malnourished children of the FSL - Support group however, there is a need to carry out more specific researches to understand the causal framework of malnutrition in Sanghar district and rest of the hotspots in the country. The average weight gain of the malnourished children was almost the same after 90 days of the follow up of the children from both the groups.

Conclusion: The findings of the study are strongly suggestive of integration of nutrition specific interventions as treatment of choice with better results when combined with nutrition sensitive activities. The subject should further be studied to have evidence based programming in the country for long term follow up in cohorts and to assess resilience to develop malnutrition among both of the groups.
\end{abstract}

\section{Introduction}

Malnutrition is one of the major issues in the developing and underdeveloped world from the point of view of public health as various sources confirm that more than 50 percent of the mortality among $U 5$ years of children is contributed to malnutrition. Lancet series on maternal and child nutrition in 2008 was an eye opener for the whole of world as 45 percent of the U 5 year mortality was attributable to malnutrition [1].

Situation is Pakistan is worse than the neighboring countries. The National Nutrition Survey 2011 (NNS 2011) is suggesting that Pakistan is suffering from double burden of acute and chronic malnutrition coupled with emerging cases of over nutrition or obesity [2].

Malnutrition is a complicated phenomenon and as one can see from the conceptual framework of malnutrition (Figure 1) that malnutrition is immediately caused by; lack of food and prevailing disease condition, underlying causes as food security and quality, care resources and practices, health services and water, sanitation, hygiene (WASH) practices, and basic causes of malnutrition as; institutions, political and ideological framework, economic structure, resources, environment, technology, and people. 


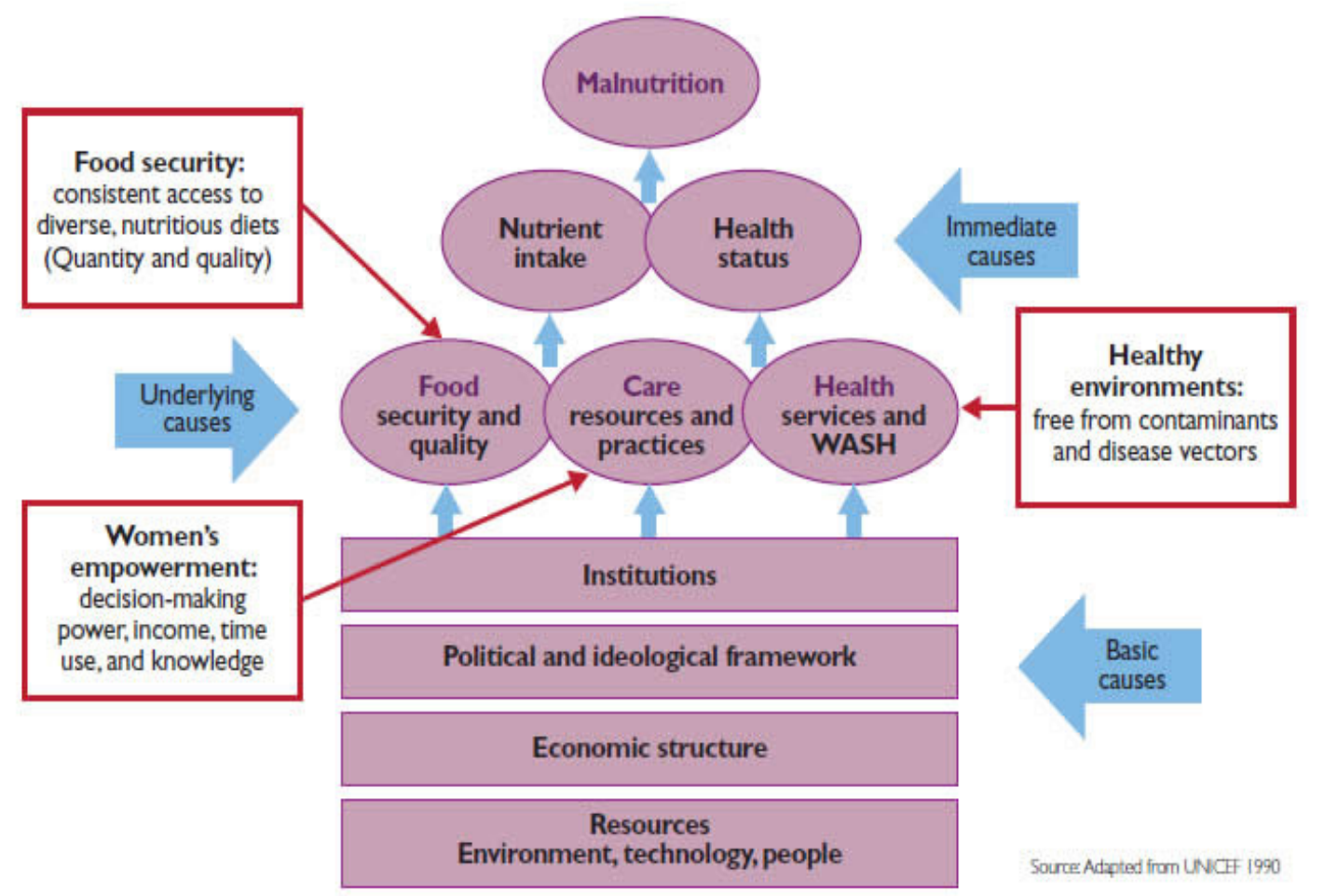

Figure 1: Conceptual Framework of Malnutrition [3]

One of the global movement to address malnutrition in developing and under developed countries is the "Scaling Up Nutrition" (SUN) movement and Pakistan has recently became signatory to it (2013) realizing the extremely high figures of stunting and wasting (NNS 2011). The main purpose of the movement is address malnutrition with global and national political commitment to adopt multisectoral approaches and policies from various sectors like business network, academia and research network, and civil society alliance [4]. Global advocacy movements and with the efforts of World Health Organization and recommendations from the Lancet Series, Pakistan has realized the need of investing in nutrition of the nation with focus on U 5 years children and integrated and standalone Planning Commission Documents (PC1) have been developed in all of the provinces including Azad Jammu and Kashmir (AJK) and Gilgit-Baltistan (GB). The purpose of these PC 1s is to integrate nutrition programming with health sectors of the provinces and provide lifesaving nutrition specific services and promote preventive measures to avoid malnutrition in the communities. Similarly for the purpose of multisectoal approaches SUN secretariat has been established at federal level within the Planning Commission of Pakistan and under the SUN secretariat other networks like business network, civil society alliance, and Academia and Research networks are also in the process of establishment. The same will be translating to provincial level and the process has been initiated under the leadership of SUN secretariat. Malnutrition in Pakistan is a key concern and the provincial government of AJK, Balochistan, GB, Khyber Pakhtoonkhwa (KP), Punjab, Sindh, under the leadership of federal has starting investing in malnutrition and civil society organizations are piloting small scale projects to provide lifesaving nutrition specific and sensitive interventions for the immediate assistance in the flood hit areas.

Save the Children in close coordination and collaboration with provincial department of health and Nutrition Cell, Sindh is implementing project in the drought and flood affected district Sanghar [5]. The assistance is provided through nutrition sensitive interventions focusing on health and nutrition education, WASH and coupled with monitory support to the poor households to improve food security; and nutrition specific interventions as per the WHO guidelines of Community Based Management of Acute Malnutrition (CMAM), which is the treatment component of the U 5 years children and Pregnant and Lactating Women (PLW). The project is designed on the multisectoral approaches to address malnutrition [6,7].

Under the subject project a total of 3,350 U 5 year children were enrolled in the OTPs for the treatment of Severe Acute Malnutrition. Out of these children, household of 367 were provided with FSL support in addition to the treatment as the economic status of these households was very poor and per the predefined criteria of the project. The FSL support was provided in terms of monthly monetary incentives of PKR 1,800/- per month for 6 months.

Nutrition programming in Sindh and similarly in Pakistan are very recent and the current research is carried out to observe various aspects of the nutrition support through standalone treatment services and services with additional support in terms of improving household food security. The aim of the research is evaluate the project implemented by Save the Children in Saghar and assess the nutrition status of the $U 5$ year children with treatment support under CMAM protocols and the same among $U$ 5 children receiving treatment and support to improve house hold food security. The research is based on the secondary data analysis of the project database. 
Though very limited research is available on the subject however, many success stories could be found around the world resulting in better nutrition outcomes.

As evident from the conceptual framework of malnutrition there is a need to adopt multisectoral and multidimensional approaches to address malnutrition. It is worth mentioning that nutrition is key to human development and reduction in hunger is the top priority of MDGs. The same is on top of the post development agenda i.e. Sustainable Development Goals (SDGs) 2015. Despite the realization of multisectoral approaches to tackle malnutrition, there is little evidence available to effectively link the evidence with best practices and contribution of various factors in reducing malnutrition.

In Sub Sahara Africa, Senegal is the country close to achieve the Millennium Development Goal (MDG) for nutrition through its multisectoral Nutrition Enhancement Programme (NEP) through close coordination and collaboration among the ministries, donors, and NGOs under the umbrella of the prime minister office and public private partnership for the reduction in malnutrition [8].

Almost the same approach was also adopted in Colombia with development of network, focusing on complementary feeding, health services, agriculture, and education with involvement of government and public private initiatives to reduce malnutrition [9].

Similarly realizing the need for multisectoral approaches to address malnutrition, a global movement "Scaling Up Nutrition (SUN)" has been launched globally with the motive that food and adequate nutrition is a right for everyone. SUN is a country led initiative and it is based on the approach of right for food and adequate nutrition for everyone. Currently 54 countries have joined the SUN movement, including Pakistan.

In a report developed by World Bank, it was realized the MDG related to nutrition was ignored with no or poor investment in nutrition and poor political commitment with resultantly legging behind in all of the MDGs [10].

A case study from Peru in 2006 shows that nutrition specific and sensitive interventions in combination with political commitment resulted in the four percent annual reduction in stunting in the prioritized vulnerable areas.

A report developed by Food And Nutrition Technical Assistance (FANTA) and United States Agency for International Development (USAID) in April 2014 highlight that addressing malnutrition is Asia is crucial due to [11]:

- Asia is leading the world with the highest number of under 5 years children with stunting, wasting, or under weight

- The burden of malnutrition in Asia may refrain the world to achieve MDGs and subsequently the SDGs

- Maternal under nutrition is also highly prevalent in Asia with high rates of low birth weight and intrauterine growth retardation

The same report highlight the key issues linked to malnutrition in seven Southeast Asian countries namely Burma, Cambodia, Indonesia, Laos, Philippines, Timor Leste, and Vietnam as [12]:

- Low intake of iron, Zink, folate, and Vitamin A by women

- Short duration of exclusive breastfeeding

- Around half of the newborn are not supported for early initiation of breastfeeding

- Low level of iron supplementation in pregnancy

- Around $20 \%$ of the households are food insecure

- Poor water, sanitation, and hygiene practices coupled with poor access to safe drinking water

- Low level of women education

- Poor governance, insecurity, and political instability

- Poverty

Pakistan has recently joined the Scaling Up Nutrition (SUN) movement and established national level secretariat under the umbrella of Ministry of National Health Services Regulation and Coordination (MoNHSR\&C) and planning commission by realizing the need to address the alarming level of acute and chronic malnutrition and high level of food insecurity in the country through multisectoral approaches. Similarly at national Civil Society Organization's Alliance has also been established for the wide scale advocacy at national and provincial level [13]. Along with the mentioned initiative SUN Business Network has also been established to involve the private sector as a holistic approach to address malnutrition through food fortification mainly [14].

In an effort to address malnutrition, provincial level strategies in the form of Integrated PC1 for the integration of nutrition component in the services delivery for healthcare have been approved in all of the provinces. Along with the efforts carried out by the government all of the UN agencies and International NGOs are working closely with the national government and provincial Department of Health to carry out multisectoral interventions to address malnutrition through implementation of CMAM, FSL support, and WASH interventions [15].

\section{Methodology}

The research is based on the secondary data analysis of the project database of the enrolled children in the programme. As per the project database a total 3,350 U 5 year children are enrolled in the treatment for acute malnutrition and out these children, household of 374 enrolled children were also provided with conditional cash support to purchase food to improve food security 
of the household. These households were identified on the basis of poverty score used to assess poverty and food insecurity. The database was further cleaned and only those children were selected who have completed treatment. After cleaning of the data 337 children with FSL support were isolated. The children who received only treatment support were cleaned also and only 3000 children who completed treatment were isolated. Out of 3000 children, randomly 346 children were selected.

\section{Inclusion criteria}

1. All of the programme beneficiaries (Under 5) who have completed treatment as per the CMAM protocols

2. All the beneficiaries getting FSL support

\section{Exclusion criteria}

1. All the screened children with severe malnutrition and other medical conditions were excluded from the study

\section{Results}

The analysis of the data shows that weight gain in the FSL support group was faster as compare to those children who were not provided with FSL support.

\section{Result: Treatment outcome after one week of the admission in OTP}

All the enrolled children from both of the groups were followed up on the next week for the assessment of weight gain through MUAC and provision of RUTF for the next week (Table 1 and 2).

\begin{tabular}{|l|l|l|l|}
\hline \multirow{2}{*}{ Description } & \multicolumn{2}{|l|}{ Type of Support } & \multirow{2}{*}{ Total } \\
\cline { 2 - 3 } & Non FSL Support & FSL Support & \\
\hline MUAC at Admission, & 346 & 337 & 683 \\
SAM $(<11.5 \mathrm{~cm})$ & $50.8 \%$ & $49.2 \%$ & $100 \%$ \\
\hline \multirow{2}{*}{ Exits (MUAC $>11.5 \mathrm{~cm})$} & 0 & 0 & 0 \\
\hline \multirow{2}{*}{ Total } & $0 \%$ & $0 \%$ & $0 \%$ \\
\hline
\end{tabular}

Table 1: Follow up of the SAM children after a week through MUAC

\begin{tabular}{|l|l|l|l|}
\hline \multirow{2}{*}{ Description } & \multicolumn{2}{|l|}{ Type of Support } & \multirow{2}{*}{ Total } \\
\cline { 2 - 3 } & Non FSL Support & FSL Support & \\
\hline $\begin{array}{l}\text { MUAC at Week-2, SAM } \\
<11.5 \mathrm{~cm})\end{array}$ & 239 & 190 & 429 \\
\hline \multirow{2}{*}{ Exits (MUAC $>11.5 \mathrm{~cm})$} & $55.7 \%$ & $44.3 \%$ & $100 \%$ \\
\hline \multirow{2}{*}{ Total } & $47.1 \%$ & 117 & 221 \\
& 346 & $52.9 \%$ & $100 \%$ \\
\hline Chi Square Value & $50.7 \%$ & 337 & 683 \\
\hline
\end{tabular}

Table 2: Follow up visit after a month of the children from both of the groups through MUAC measurement

\section{Discussion}

Food insecurity is among the underlying cause of malnutrition and the main reason for food insecurity of the households or communities is poverty, disasters, wars, general insecurity, and market failures due to above or other reasons. The world has adopted various approaches like SUN movement [16,17], Zero Hunger Programme, Multisectoral Strategies, to address acute and chronic malnutrition for the development in health and economically. Various evidences prove that improved nutrition status lead to increased productivity and economic growth. Investment in nutrition has proved to increase GDP whereas no investment in nutrition returned in loss in GDP [18]. In the literature review we have global, regional, and national evidences suggestive multisectoral approaches to address malnutrition and have successfully achieved the results. Similarly the Lancet Series on Maternal and Child Nutrition is also advocating for the global leaders to address all types of malnutrition through the sustainable Development Goals of 2016-2025 [19]. Research shows that 53 percent of the U 5 year children mortality can be attributed to malnutrition [20]. Though there is no direct link of malnutrition with mortality except in famine, still malnutrition make the organism susceptible to various health risks. Malnutrition is affecting health of all age group of the population however; infant and children are considered to be the most vulnerable groups due to high energy requirement for growth and development. Similarly pregnant and lactating women are also included in risk group for malnutrition due to feeding of their children or increased energy requirements due to pregnancy. 
Sindh has a complex socio-economic and diverse weather conditions and in district Sanghar, we have the arid lands with production of fruits, staple foods, and cash crops however, there is deserted area also part of the district. Similarly the economic disparities are also there [21]. As a result the landlords are enjoying good life and the Haaris (who work in the lands as labor) are struggling for the basic necessities of life. Similarly the water, sanitation, and hygiene conditions are worst in Sindh, 60 percent of population is lacking access to clean drinking water, and more than 50 percent of the population is practicing open defecation [22]. The same study also suggests that maternal education is directly proportional to the nutrition status of her children. As per the results of National Nutrition Survey (NNS - 2011), in Sindh more than 60 percent of the households are food insecure with Global Acute Malnutrition (GAM) rates of 17.2 percent, which is above the WHO criteria of emergency threshold of 15 percent. The government of Sindh is working on policies to improve food security in the province however, the implementation will take time.

The distribution of health services is also not equal throughout Pakistan [23] including in Sindh and particularly in district Sanghar. Access to healthcare is a major challenge for the poor population and out of pocket spending of health is highest in Pakistan, estimated 72\% [23]. In case of any major health issues, the communities are compelled to sell their assets or take loan on high interest rates to tackle with the situation and resultantly facing severe food insecurity [24].

Disease condition and lack of food are the important and major barriers for better nutrition of the society. The same are actually essential components of the viscous cycle of poverty and there is a high need to invest in these two sectors on emergency basis to save lives and to avoid generations of malnourished in the country there is a need to invest in stunting prevention (chronic malnutrition) through promotion of Infant and Young Child Feeding (IYCF), maternal education, access to healthcare, water and sanitation \& hygiene, and income generation.

\section{Conclusions}

The findings of the research are strongly suggestive of multisectoral and multidirectional approaches to address the critical issue of malnutrition in the country. There is evident improvement in nutrition status of the U 5 year children among the FSL support group who are from the economically deprived households in comparison to the Non FSL support group. The monetary incentives in terms of FSL support was very less i.e. PKR 1,800/- per month for 6 months however, with the existing structure of Benazir Income Support Programme (BISP), Pakistan Bait - ul - Maal, and other social safety nets can be easily mobilized and sensitized to identify the economically deprived households and provide support for the improvement of their food security and livelihood. The same approaches to target the vulnerable households in the worst food insecure areas can be targeted through social insurance which is going to be launched soon in Pakistan.

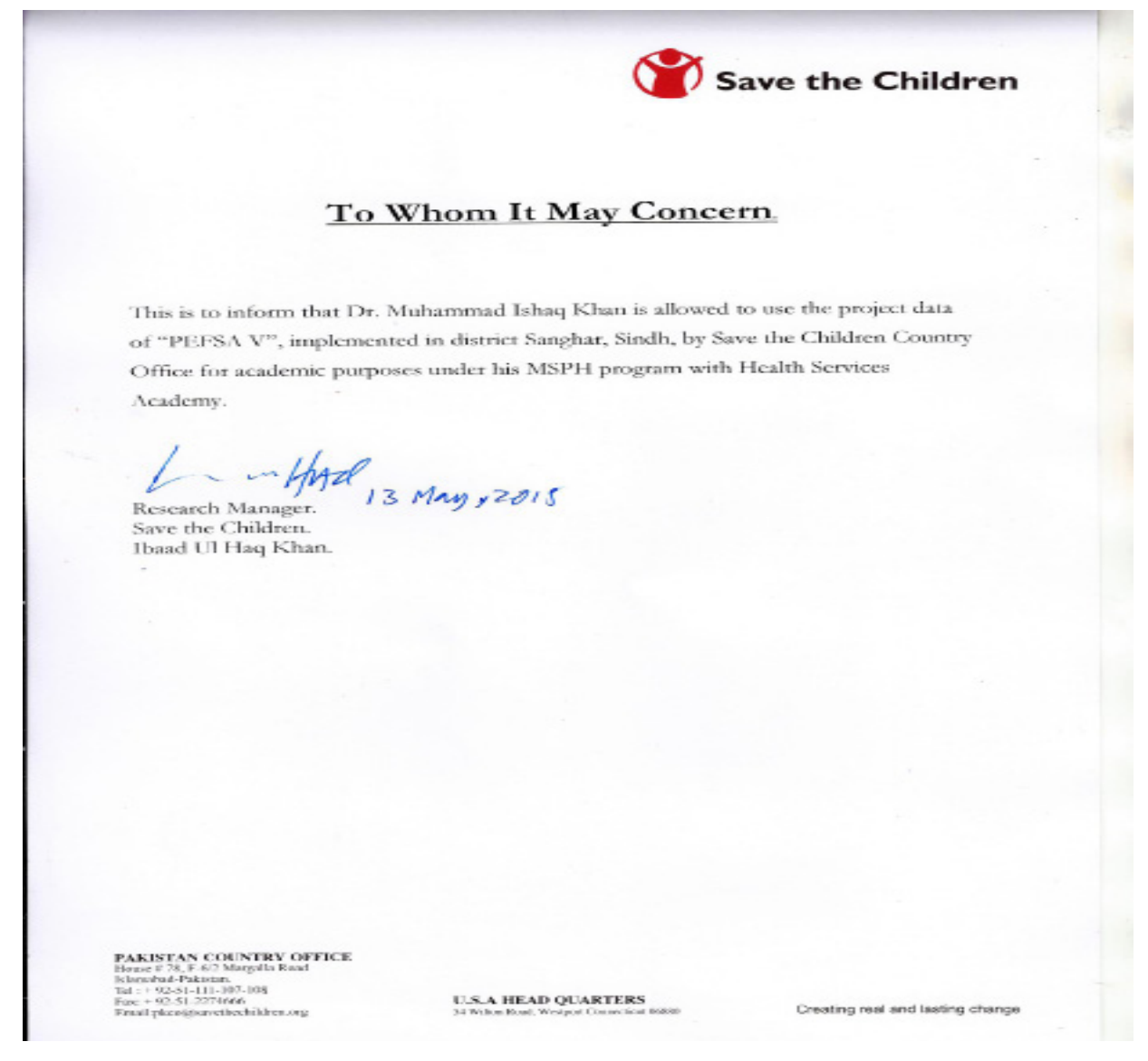


The current study is a minor effort to understand and observe if there is any improvement in the treatment of $U 5$ year children with FSL support; there is a need to carry out more researches to largely understand the causal factors of malnutrition in Pakistan and there is a need to develop cause specific strategic plan to address malnutrition in the country.

Based on the findings of the results of the study and lesson learnt from the project implementation, below key points are recommend for the way forward:

- Identify the hot spot areas of malnutrition in Pakistan through nutrition specific surveys and studies like SMART

- Identify the economically deprived households in the hotspot areas of Pakistan

- Develop coordination mechanism among all the social safety nets programmes

- Develop strategies to target the vulnerable households for food security and treatment of malnutrition

- Sectoral coordination at district level

- Behavior Change Communication (BCC) for healthy life style and nutrition through local available electronic and print media

\section{References}

1. Maternal, Group CNS (2013) Executive Summary of The Lancet Maternal and Child Nutrition Series. Oxford, UK: Elsevier.

2. National Nutrition Survey (2011) Pakistan.

3. Conceptual Framework of malnutrition UNICEF (1999).

4. Planning Commission of Pakistan, Chief Nutrition Mr. Aslam Shaheen.

5. Formative Research on Behavior Change Communication, Shikarpur, Save the Children.

6. Pakistan Emergency Food Security Alliance V (PEFSA V) 2014-15.

7. Women, infant, and children Improved Nutrition (WINS) Shikarpur, Save the Children.

8. Garrett J, Natalicchio M (2011) Working multisectorally in nutrition: Principles, practices, and case studies.

9. Nutrition policy-Colombia-Antioquia-Case studies.

10. Improving Nutrition Through Multisectoral Approaches.

11. Overview of the Nutrition Situation in Seven Countries in Southeast Asia.

12. Overview of the Nutrition Situation in Seven Countries in Southeast Asia Camila Chaparro, Lesley Oot, and Kavita Sethuraman April 2014.

13. Ministry of planning, Islamabad, Pakistan.

14. MoNHSR\&C, Islamabad, Pakistan.

15. Provincial Nutrition Cell, Sindh.

16. Scaling Up Nutrition Movement.

17. Scaling Up Nutrition. A framework for action.

18. Why invest in Nutrition, World Bank 2003.

19. Executive Summary of the Lancet on Maternal and Child Nutrition Series, 2008.

20. Blakely T, Hales S, Woodward A (2004) Socioeconomic status: assessing the distribution of health risks by socioeconomic position at national and local levels. Geneva, World Health Organization.

21. Knowledge Attitude and Practices Survey, Sanghar, Sindh by Save the Children in August 2014.

22. Comprehensive Assessment of Sindh (CAS), Save the Children, May 2015.

23. Pakistan Demographic and Health Survey 2012-13.

24. Comprehensive Assessment of Sindh (CAS) for nutrition causal analysis, May 2015.

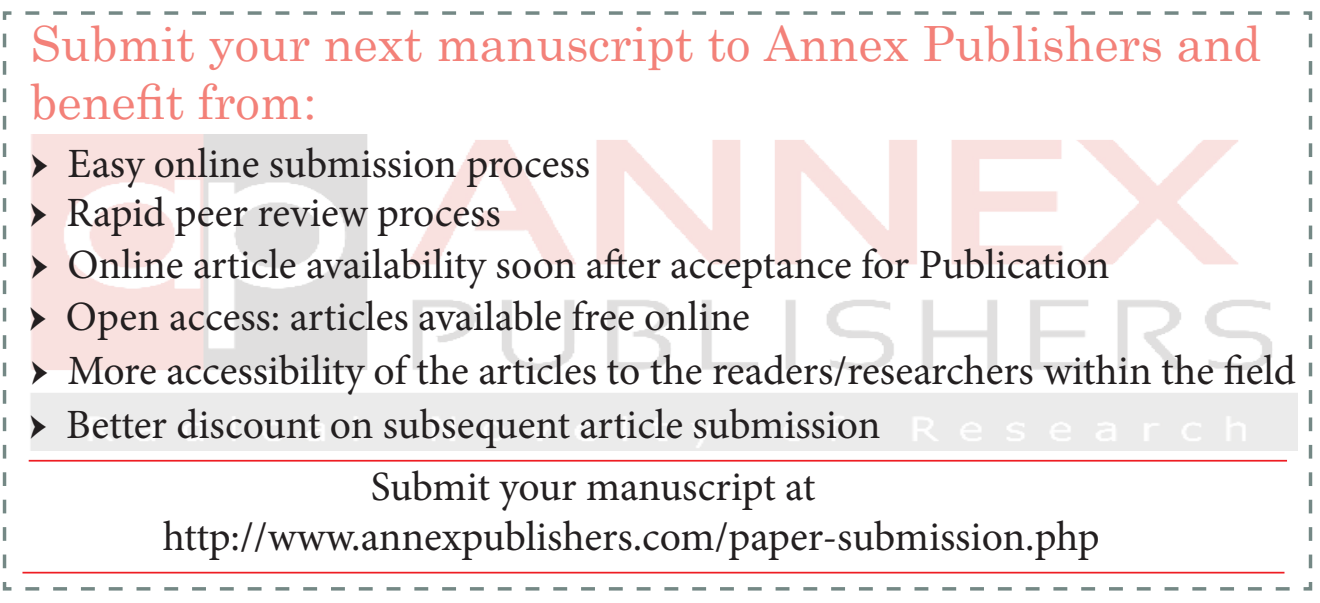

